\title{
On Semi-implicit Splitting Schemes for the Beltrami Color Image Filtering
}

\author{
Guy Rosman • Lorina Dascal · Xue-Cheng Tai • \\ Ron Kimmel
}

Published online: 15 January 2011

(c) The Author(s) 2011. This article is published with open access at Springerlink.com

\begin{abstract}
The Beltrami flow is an efficient nonlinear filter, that was shown to be effective for color image processing. The corresponding anisotropic diffusion operator strongly couples the spectral components. Usually, this flow is implemented by explicit schemes, that are stable only for very small time steps and therefore require many iterations. In this paper we introduce a semi-implicit Crank-Nicolson scheme based on locally one-dimensional (LOD)/additive operator splitting (AOS) for implementing the anisotropic Beltrami operator. The mixed spatial derivatives are treated explicitly, while the non-mixed derivatives are approximated
\end{abstract}

This research was partly supported by United States-Israel Binational Science Foundation grant No. 2004274, ISF grant No. 1551/09, by ONR grant No. N00014-06-1-0978, the Ministry of Science grant No. 3-3414 and by Rubin Scientific and Medical Fund and the Elias Fund for Medical Research. The research is also supported by MOE (Ministry of Education) Tier II project T207N2202 and IDM project NRF2007IDMIDM002-010.

G. Rosman (凶) · L. Dascal · R. Kimmel

Department of Computer Science, Technion, Israel Institute

of Technology, Haifa 32000, Israel

e-mail: rosman@cs.technion.ac.il

L. Dascal

e-mail: lorina@cs.technion.ac.il

R. Kimmel

e-mail: ron@cs.technion.ac.il

X.-C. Tai

Department of Mathematics, University of Bergen, Johannes Brunsgate 12, 5007 Bergen, Norway

e-mail: tai@math.uib.no

X.-C. Tai

Division of Mathematical Sciences, School of Physical

Mathematical Sciences, Nanyang Technological University,

Singapore, Singapore

e-mail: xctai@ntu.edu.s in an implicit manner. In case of constant coefficients, the LOD splitting scheme is proven to be unconditionally stable. Numerical experiments indicate that the proposed scheme is also stable in more general settings. Stability, accuracy, and efficiency of the splitting schemes are tested in applications such as the Beltrami-based scale-space, Beltrami denoising and Beltrami deblurring. In order to further accelerate the convergence of the numerical scheme, the reduced rank extrapolation (RRE) vector extrapolation technique is employed.

Keywords Splitting methods $\cdot$ Beltrami flow $\cdot$ Image denoising $\cdot$ Diffusion

\section{Introduction}

Nonlinear diffusion filters based on partial differential equations (PDEs) have been extensively used in the last decade for different tasks in image processing. Their efficient implementation requires designing numerical schemes in which the issues of accuracy, stability, and computational cost all play important roles.

The Beltrami image flow is an example of a nonlinear filter, that is efficient for color image processing. It treats the image as a 2-D manifold embedded in a hybrid spatialfeature space. Minimization of the image area surface yields the Beltrami flow. The corresponding diffusion operator is anisotropic and strongly couples the spectral components. Due to its anisotropy and non-separability, so far there is no efficient implicit, nor operator-splitting-based numerical scheme for the partial differential equation that describes the Beltrami flow in color. Usual discretizations of this filter are based on explicit schemes, that limit the time step and therefore result in a large number of iterations. In [7] an acceler- 
ation technique based on the RRE (reduced rank extrapolation) algorithm was proposed in order to speed-up the slow convergence of the explicit scheme.

As an alternative to the explicit scheme, an approximation using the short time kernel of the Beltrami operator was suggested in [26]. Although unconditionally stable, this method is still computationally demanding, since computing the kernel involves geodesic distance computation around each pixel.

The bilateral filter, which can be shown to be an Euclidean approximation of the Beltrami kernel, was studied in different contexts (see [2, 4, 10, 22, 23, 27]), and in [18] signal processing acceleration methods were proposed for efficient evaluation of this filter. Recently, a related filter, the nonlocal means filter, was proposed in [6] and shown to be useful in denoising of gray-scale and color images. Its application to fast video processing and surface smoothing was shown in $[14,33]$.

In this paper we propose to approximate the system of nonlinear coupled equations given by the Beltrami flow using a semi-implicit finite difference scheme based on operator splitting. Historically, additive operator splitting (AOS) schemes were first developed for (nonlinear elliptic/parabolic) monotone equations and Navier-Stokes equations $[12,13]$. In image processing applications, the AOS scheme was found to be an efficient way for approximating the Perona-Malik filter [29], especially if symmetry in scale-space is required. The AOS scheme is first order in time, semi-implicit, and unconditionally stable with respect to its time-step [13, 29]. In the early 1950's (see [19]) the alternating-direction method (ADI) was introduced, and in [31] the LOD (locally one-dimensional) splitting method was proposed. The LOD scheme and other multiplicative splitting methods were employed in the context of nonlinear diffusion image filtering in [5]. We stress that the main characteristic of this class of equations, which allows splitting, is local isotropy. However, in the case of the anisotropic Beltrami operator, the main difficulty in splitting stems from the presence of the mixed derivatives. To overcome this problem, we suggest to construct the following semi-implicit scheme; the spatial mixed derivatives are discretized explicitly at the current time step $n \Delta t$, while those that do not contain mixed derivatives are approximated using an average of two levels of time steps: $n \Delta t$ and $(n+1) \Delta t$ (Crank-Nicolson scheme). Preliminary results concerning this scheme were presented in a previous conference paper [8]. We now proceed to extend these with both a theoretical analysis of the stability and new experimental results.

Regarding the suggested scheme, as our equations are nonlinear, a stability proof of the resulting finite difference equations is a non-trivial task. We propose accordingly to use the von-Neumann analysis of the suggested scheme under the assumption of constant coefficients (for both cases of scale space and denoising). This analysis shows that for this simple case, the LOD splitting scheme is unconditionally stable. Furthermore, our numerical experiments indicate that the LOD and the AOS splitting schemes for the nonlinear Beltrami color filter also display a stable behavior. We demonstrate the efficiency and stability of the splitting in applications such as: Beltrami-based scale space and Beltrami-based denoising and deblurring. In order to further expedite the LOD/AOS splitting schemes, we show how to speed-up their convergence by using the RRE (reduced rank extrapolation) technique. The RRE method was introduced by Mešina and Eddy $[9,17]$ to speed-up the convergence of general sequences of vectors without explicit knowledge of the sequence generator. This technique was applied in [7] in order to speed up the slow convergence of the standard explicit scheme for the Beltrami color flow. In this paper we show that in applications such as scale-space and denoising of color images, the semi-implicit LOD/AOS schemes can also be accelerated using the RRE technique.

This paper is organized as follows: In Sect. 2 we briefly summarize the Beltrami framework. In Sect. 3 we review general semi-implicit splitting operator schemes for the linear heat equation. In Sect. 4 we propose a semiimplicit splitting scheme for the anisotropic Beltrami operator, based on the LOD/AOS schemes. We provide the vonNeumann stability analysis of the LOD-based scheme which is valid in the case where the coefficients are constants. In Sect. 5 we demonstrate the efficiency and stability of the LOD/AOS splitting schemes for Beltrami-based scale-space and Beltrami-based denoising. Furthermore, we propose to accelerate the LOD/AOS schemes using the RRE technique. Section 6 concludes the paper.

\section{The Beltrami Framework}

Let us briefly review the Beltrami framework for non-linear diffusion in computer vision $[11,24,25,32]$. We represent images as embedding maps of a Riemannian manifold in a higher dimensional space. We denote the map by $U: \Sigma \rightarrow M$, where $\Sigma$ is a two-dimensional surface, with $\left(\sigma^{1}, \sigma^{2}\right)$ denoting coordinates on it. $M$ is the spatial-feature manifold, embedded in $\mathbb{R}^{d+2}$, where $d$ is the number of image channels. For example, a gray-level image can be represented as a $2 \mathrm{D}$ surface embedded in $\mathbb{R}^{3}$. The map $U$ in this case is $U\left(\sigma^{1}, \sigma^{2}\right)=\left(\sigma^{1}, \sigma^{2}, I\left(\sigma^{1}, \sigma^{2}\right)\right)$, where $I$ is the image intensity. For color images, $U$ is given by

$U\left(\sigma^{1}, \sigma^{2}\right)=\left(\sigma^{1}, \sigma^{2}, I^{1}\left(\sigma^{1}, \sigma^{2}\right), I^{2}\left(\sigma^{1}, \sigma^{2}\right), I^{3}\left(\sigma^{1}, \sigma^{2}\right)\right)$, where $I^{1}, I^{2}, I^{3}$ are the three components of the color vector.

Next, we choose a Riemannian metric on this surface, $g$, with elements denoted by $g_{i j}$. The canonical choice of coordinates in image processing is Cartesian (we denote them 
here by $x^{1}$ and $x^{2}$ ). For such a choice, which we follow in the rest of the paper, we identify $\sigma^{1}=x^{1}$ and $\sigma^{2}=x^{2}$. In this case, $\sigma^{1}$ and $\sigma^{2}$ are the image coordinates. We denote the elements of the inverse of the metric by superscripts $g^{i j}$, and the determinant by $g=\operatorname{det}\left(g_{i j}\right)$. Once images are defined as embedding of Riemannian manifolds, it is natural to look for a measure on this space of embedding maps.

Denote by $(\Sigma, g)$ the image manifold and its metric, and by $(M, h)$ the space-feature manifold and its metric. Then, the functional $S[U]$ assigns a real number to a map $U: \Sigma \rightarrow M$,

$S\left[U, g_{i j}, h_{a b}\right]=\int d^{s} \sigma \sqrt{g}\|d U\|_{g, h}^{2}$,

where $s$ is the dimension of $\Sigma, g$ is the determinant of the image metric, and the range of indices is $i, j=$ $1,2, \ldots \operatorname{dim}(\Sigma)$ and $a, b=1,2, \ldots \operatorname{dim}(M)$. The integrand $\|d U\|_{g, h}^{2}$ is expressed in a local coordinate system by $\|d U\|_{g, h}^{2}=\left(\partial_{x_{i}} U^{a}\right) g^{i j}\left(\partial_{x_{j}} U^{b}\right) h_{a b}$. This functional, for $\operatorname{dim}(\Sigma)=2$ and $h_{a b}=\delta_{a b}$, was first proposed by Polyakov [20] in the context of high energy physics, in the theory known as string theory. The elements of the induced metric for color images with Cartesian color coordinates are

$$
\begin{aligned}
G & =\left(g_{i j}\right) \\
& =\left(\begin{array}{cc}
1+\beta^{2} \sum_{a=1}^{3}\left(U_{x_{1}}^{a}\right)^{2} & \beta^{2} \sum_{a=1}^{3} U_{x_{1}}^{a} U_{x_{2}}^{a} \\
\beta^{2} \sum_{a=1}^{3} U_{x_{1}}^{a} U_{x_{2}}^{a} & 1+\beta^{2} \sum_{a=1}^{3}\left(U_{x_{2}}^{a}\right)^{2}
\end{array}\right),
\end{aligned}
$$

where a subscript of $U$ denotes a partial derivative and the parameter $\beta>0$ determines the ratio between the spatial and spectral (color) distances. Using standard methods in calculus of variations, the Euler-Lagrange equations with respect to the embedding (assuming Euclidean embedding space) are

$$
0=-\frac{1}{\sqrt{g}} h^{a b} \frac{\delta S}{\delta U^{b}}=\underbrace{\frac{1}{\sqrt{g}} \operatorname{div}\left(D \nabla U^{a}\right)}_{\Delta_{g} U^{a}},
$$

where the diffusion matrix is

$$
D=\sqrt{g} G^{-1} \text {. }
$$

Note that we can write

$$
\operatorname{div}(D \nabla U)=\sum_{q, r=1}^{2} \partial_{x_{q}}\left(d_{q r} \partial_{x_{r}} U\right) .
$$

The operator that acts on $U$ is the natural generalization of the Laplacian from flat spaces to manifolds. It is called the Laplace-Beltrami operator, and denoted by $\Delta_{g}$.

The parameter $\beta$, in the elements of the metric $g_{i j}$, determines the nature of the flow. At the limits, where $\beta \rightarrow 0$ and $\beta \rightarrow \infty$, we obtain respectively a linear diffusion flow and a nonlinear flow, akin to the TV flow [21] for the case of grey-level images (see [25] for details).

The Beltrami scale-space emerges as a gradient descent minimization process

$U_{t}^{a}=-\frac{1}{\sqrt{g}} \frac{\delta S}{\delta U^{a}}=\Delta_{g} U^{a}, \quad a=1,2,3$.

For Euclidean embedding, the functional in (1) reduces to

$$
\begin{aligned}
S(U)= & \int \sqrt{g} d x^{1} d x^{2} \\
= & \int \sqrt{1+\beta^{2} \sum_{a=1}^{3}\left|\nabla U^{a}\right|^{2}+\frac{1}{2} \beta^{4} \sum_{a, b=1}^{3}\left|\nabla U^{a} \times \nabla U^{b}\right|^{2}} \\
& \times d x^{1} d x^{2} .
\end{aligned}
$$

This geometric measure can be used as a regularization term for color image processing. In the variational framework, the reconstructed image is the minimizer of a costfunctional. This functional can be written in the following general form,

$\Psi(U)=\lambda \sum_{a=1}^{3}\left\|U^{a}-F^{a}\right\|^{2}+S(U)$,

where the parameter $\lambda$ controls the smoothness of the solution and $F$ is the given image.

The modified Euler-Lagrange equations as a gradient descent process are

$$
\begin{aligned}
U_{t}^{a} & =-\frac{1}{\sqrt{g}} \frac{\delta \Psi}{\delta U^{a}}=-\frac{2 \lambda}{\sqrt{g}}\left(U^{a}-F^{a}\right)+\Delta_{g} U^{a}, \\
a & =1,2,3 .
\end{aligned}
$$

The above equations characterize an adaptive smoothing mechanism. In areas with large gradients (edges), the fidelity term is suppressed and the regularizing term becomes dominant. At homogenous regions with low-gradient magnitude, the fidelity term takes over and controls the flow.

\section{Operator Splitting Schemes}

In this section we review standard first order accurate splitting schemes for the two-dimensional heat equation. Splitting techniques are commonly employed in solving timedependent partial differential equations. They are used in order to reduce problems in multiple spatial dimensions to a sequence of problems in one dimension, which are easier to solve. 
Consider the second order heat equation

$u_{t}=\sum_{i=1}^{2} \partial_{x_{i}}\left(\partial_{x_{i}} u\right), \quad$ in $\Omega \times[0, T]$

with suitable boundary conditions on $\partial \Omega \times[0, T]$, where $\Omega$ is a rectangle in $\mathbf{R}^{2}, \partial \Omega$ is the boundary of $\Omega$, and $[0, T]$ is the time interval $0 \leq t \leq T$.

We discretize this equation on a rectangular grid of size $N=m \times m$. The time steps are given by $t_{n}=n \Delta t, 1 \leq n \leq$ $J$ and $J \Delta t=T$. Denote by $U^{n}$ the approximate $N$ dimensional solution at the time level $n$. The semi-implicit approximation scheme can be written in vector-matrix notation

$\left(I-\Delta t \sum_{l=1}^{2} A_{l l}\left(U^{n}\right)\right) U^{n+1}=U^{n}$,

where the $N \times N$ matrix $A_{l l}$ is the finite difference approximation of the second order differential operator corresponds to the derivatives along the $l$-th coordinate axis. The main drawback of this implicit scheme is the high computational cost needed to invert the matrix $I-\Delta t \sum_{l=1}^{2} A_{l l}\left(U^{n}\right)$, because unlike the one-dimensional case, the matrix is not tridiagonal and therefore cannot be inverted in an efficient manner. In order to overcome this problem, splitting methods were proposed $[15,19]$. One of the simplest splitting schemes belonging to the class of multiplicative operator splitting schemes, is the locally one-dimensional (LOD) scheme [31]

$U^{n+1}=\prod_{l=1}^{2}\left(I-\Delta t A_{l l}\left(U^{n}\right)\right)^{-1} U^{n}$.

The LOD scheme only needs to invert some three-diagonal matrices. It is simple to implement, is unconditionally stable and it is first order accurate. However, the system matrix in (11) is not axis symmetric, a property that may be important in some cases.

If such a property is required, one could use the additive operator splitting (AOS) [13] which was actually invented for parallel implementation of splitting methods

$U^{n+1}=\frac{1}{2} \sum_{l=1}^{2}\left(I-2 \Delta t A_{l l}\left(U^{n}\right)\right)^{-1} U^{n}$.

Note that the two three-diagonal matrices can to be inverted in parallel. Even for sequential implementations, the AOS is almost as efficient as the LOD scheme; instead of multiplying the operators, one computes them independently and then average the sums of the inverse of the two matrices. We want to emphasis that the matrices for AOS use $2 \Delta t$ instead of $\Delta t$.
It is not a trivial matter to apply dimensional splitting schemes for Beltrami type of equations. Our goal is to construct a splitting scheme for the nonlinear anisotropic Beltrami operator, which would amount to inverting tridiagonal matrices, be unconditionally stable and preserve the time discretization accuracy that was obtained before the splitting.

\section{The Proposed Splitting Scheme}

In this section we propose a first order accurate operator splitting scheme for the Beltrami filter. Before splitting, we first introduce a semi-implicit approximation scheme to our equations.

A semi-implicit Crank-Nicolson scheme for an equation involving mixed derivatives can rely on the following discretization of the spatial derivatives operators: mixed derivatives are computed at time step $n \Delta t$, while the non-mixed derivatives are computed as the average of values at time steps $n \Delta t$ and $(n+1) \Delta t$. This approach for handling mixed derivatives in semi-implicit schemes for approximating linear equations has been considered in several previous works (see $[1,16,30]$ for example), including the context of image processing [28], although it was not combined with the Crank-Nicolson method in the case of [28]. In [1] a stability analysis of a splitting scheme approximating a general class of linear parabolic equations with mixed derivatives was performed. The a priori estimates of the solution were obtained by the method of energy inequalities and by assuming certain bounds of the quadratic form involved in the parabolic equation. The Crank-Nicolson based splitting scheme for the linear case presented in this paper is a particular case of the general scheme given in [1] (for $\sigma=0.5$ ). However, the stability proof we present here is based on a different and simpler method, namely the von-Neumann analysis. We furthermore accentuate that our proof relies only on the assumption of parabolicity of the equations and it does not need any assumptions on the boundedness of the quadratic form. McKee and Mitchell developed a slightly different scheme [16]. They utilized a von-Neumann analysis in their stability proof, in a similar manner to our own. Their proposed scheme, however, is different and specifically, it is not symmetric with respect to the non-mixed directional derivative operators.

We now proceed to describe our proposed scheme. First, let us refine our grid notations. We work on the rectangle $\Omega=(0,1) \times(0,1)$, which we discretize by a uniform grid of $m \times m$ pixels, such that $x_{i}=i \Delta x, y_{j}=j \Delta y, t_{n}=n \Delta t$, where $1 \leq i \leq m, 1 \leq j \leq m, n=1,2, \ldots, J$ and $J \Delta t=T$. Let the grid size be $\Delta x=\Delta y=h=\frac{1}{m-1}$.

For each channel $U^{a}, a=1,2,3$ of the color vector, we define the discrete approximation $\left(U^{a}\right)_{i j}^{n}$ by

$\left(U^{a}\right)(i \Delta x, j \Delta y, n \Delta t)=\left(U^{a}\right)_{i j}^{n} \approx U^{a}(i \Delta x, j \Delta y, n \Delta t)$. 
We impose von-Neumann boundary condition, and initially set $U^{a}$ to be our initial data image.

\subsection{LOD/AOS Scheme for the Beltrami Scale-Space}

We approximate the Beltrami filter given in (5) by the following semi-implicit Crank-Nicolson scheme:

$$
\begin{aligned}
& \frac{\left(U^{a}\right)^{n+1}-\left(U^{a}\right)^{n}}{\Delta t} \\
& =\frac{1}{\sqrt{g^{n}}}\left(\frac{1}{2} \sum_{l=1}^{2} A_{l l}^{n}\left(U^{a}\right)^{n+1}+\frac{1}{2} \sum_{l=1}^{2} A_{l l}^{n}\left(U^{a}\right)^{n}\right. \\
& \left.\quad+\sum_{q=1}^{2} \sum_{r \neq q} A_{q r}^{n}\left(U^{a}\right)^{n}\right),
\end{aligned}
$$

where $U^{a}$ is the $N$-dimensional vector denoting one of the components of the color vector, and $A_{q r}^{n}$ is a central difference approximation of the operator $\partial_{x_{q}}\left(d_{q r} \partial_{x_{r}}\right)$ at time step $n$.

After rearranging terms, we have

$$
\begin{aligned}
\left(U^{a}\right)^{n+1} & \\
= & \left(I-\frac{\Delta t}{2 \sqrt{g^{n}}} \sum_{l=1}^{2} A_{l l}^{n}\right)^{-1} \\
& \times\left(I+\frac{\Delta t}{\sqrt{g^{n}}} \sum_{q=1}^{2} \sum_{r \neq q} A_{q r}^{n}+\frac{\Delta t}{2 \sqrt{g^{n}}} \sum_{l=1}^{2} A_{l l}^{n}\right) \\
& \times\left(U^{a}\right)^{n},
\end{aligned}
$$

which can also be written as

$$
\begin{aligned}
& \left(U^{a}\right)^{n+1} \\
& =\left(I-\frac{\Delta t}{2} \sum_{l=1}^{2} \bar{A}_{l l}^{n}\right)^{-1} \\
& \quad \times\left(I+\Delta t \sum_{q=1}^{2} \sum_{r \neq q} \bar{A}_{q r}^{n}+\frac{\Delta t}{2} \sum_{l=1}^{2} \bar{A}_{l l}^{n}\right) \\
& \quad \times\left(U^{a}\right)^{n},
\end{aligned}
$$

where

$$
\begin{array}{ll}
\bar{A}_{11}=\frac{1}{\sqrt{g}} \partial_{x}\left(A \partial_{x}\right), & \bar{A}_{22}=\frac{1}{\sqrt{g}} \partial_{y}\left(C \partial_{y}\right), \\
\bar{A}_{12}=\frac{1}{\sqrt{g}} \partial_{x}\left(B \partial_{y}\right), & \bar{A}_{21}=\frac{1}{\sqrt{g}} \partial_{y}\left(B \partial_{x}\right),
\end{array}
$$

and the functions $A, B, C$ are the corresponding elements of the diffusion matrix associated with the Beltrami flow,

$$
D=\sqrt{g} G^{-1}=\left(\begin{array}{ll}
A(\nabla U) & B(\nabla U) \\
B(\nabla U) & C(\nabla U)
\end{array}\right)
$$

Again, this semi-implicit scheme still has a major drawback. At each iteration one needs to solve a large linear system whose matrix of coefficients is not tridiagonal and thus costly. Instead, we employ the LOD splitting scheme

$$
\begin{aligned}
\left(U^{a}\right)^{n+1}= & \left(I-\frac{\Delta t}{2} \bar{A}_{22}\right)^{-1}\left(I-\frac{\Delta t}{2} \bar{A}_{11}\right)^{-1} \\
& \times\left[\left(I+\frac{\Delta t}{2} \bar{A}_{11}\right)\left(I+\frac{\Delta t}{2} \bar{A}_{22}\right)\right. \\
& \left.+\Delta t \sum_{q=1}^{2} \sum_{r \neq q} \bar{A}_{q r}^{n}\right]\left(U^{a}\right)^{n}
\end{aligned}
$$

or the AOS scheme, that reads,

$$
\begin{aligned}
\left(U^{a}\right)^{n+1}= & \frac{1}{2}\left[\left(I-\Delta t \bar{A}_{22}\right)^{-1}+\left(I-\Delta t \bar{A}_{11}\right)^{-1}\right] \\
& \times\left[\left(I+\frac{\Delta t}{2} \bar{A}_{11}\right)\left(I+\frac{\Delta t}{2} \bar{A}_{22}\right)\right. \\
& \left.+\Delta t \sum_{q=1}^{2} \sum_{r \neq q} \bar{A}_{q r}^{n}\right]\left(U^{a}\right)^{n} .
\end{aligned}
$$

The above splitting schemes are efficient because at each time step a tridiagonal matrix inversion is performed.

The system of differential equations we deal with is nonlinear. The question of theoretical stability of the LOD/AOS based nonlinear finite difference scheme is a non-trivial challenge, with theory still lagging behind common practice. Nevertheless, we can provide a von-Neumann stability analysis to a simplified case where the coefficients of the equation are set to be constants. This analysis shows that the LOD splitting scheme, at least in this over-simplistic form, is unconditionally stable. While the coefficients do not remain constant during the diffusion process in all but the simplest images, this condition is a minimal requirement in analyzing the stability of numerical schemes, and must be verified. Numerical experiments can then be performed in order to gain some empirical insight. These indicate in our case that the splitting is stable in the more general setting, as will be shown in Sect. 5.

Below we provide the von-Neumann analysis of the LOD splitting scheme. Our equations are of the form

$U_{t}^{a}=\frac{1}{\sqrt{g}} \operatorname{div}\left(D \nabla U^{a}\right)$. 
Equation (19) can be written in its general form as

$$
\begin{aligned}
U_{t}= & \frac{A}{\sqrt{g}} U_{x x}+2 \frac{B}{\sqrt{g}} U_{x y}+\frac{C}{\sqrt{g}} U_{y y} \\
& +\left(\frac{A_{x}}{\sqrt{g}}+\frac{B_{y}}{\sqrt{g}}\right) U_{x}+\left(\frac{C_{y}}{\sqrt{g}}+\frac{B_{x}}{\sqrt{g}}\right) U_{y} .
\end{aligned}
$$

In order to apply the von-Neumann analysis, the coefficients $A, B, C$ in (20) are set to be constants. Thereby, the equation is simplified to

$U_{t}=a U_{x x}+2 b U_{x y}+c U_{y y}$,

where the coefficients $a, b, c$ are now constants with $a, c>0$ and $b^{2}-a c<0$.

The Crank-Nicholson scheme with LOD splitting for approximating (19) can be written as

$$
\begin{aligned}
(I & \left.-a \frac{\Delta t}{2} \partial_{x x}\right)\left(I-c \frac{\Delta t}{2} \partial_{y y}\right) U^{n+1} \\
& =\left[\left(I+\frac{\Delta t}{2} a \partial_{x x}\right)\left(I+\frac{\Delta t}{2} c \partial_{y y}\right)+2 b \Delta t \partial_{x y}\right] U^{n},
\end{aligned}
$$

where

$$
\begin{aligned}
\partial_{x x} U_{i j}^{n} & =\frac{U_{i+1, j}^{n}-2 U_{i, j}^{n}+U_{i-1, j}^{n}}{h^{2}} \\
\partial_{y y} U_{i j}^{n} & =\frac{U_{i, j+1}^{n}-2 U_{i, j}^{n}+U_{i, j-1}^{n}}{h^{2}} \\
\partial_{x y} U_{i j}^{n} & =\frac{U_{i+1, j+1}^{n}+U_{i-1, j-1}^{n}-U_{i-1, j+1}^{n}-U_{i+1, j-1}^{n}}{4 h^{2}} .
\end{aligned}
$$

Consider a solution of the difference scheme in the form $U_{i j}^{n}=M e^{\alpha n \Delta t} e^{\sqrt{-1} i \beta h} e^{\sqrt{-1} j \gamma h}$,

where $\beta$ and $\gamma$ are constant wave numbers. We have

$$
\begin{aligned}
\partial_{x x} U_{i j}^{n} & =\frac{2}{h^{2}}(\cos (\beta h)-1) U_{i j}^{n}, \\
\partial_{y y} U_{i j}^{n} & =\frac{2}{h^{2}}(\cos (\gamma h)-1) U_{i j}^{n}, \\
\partial_{x y} U_{i j}^{n} & =-\frac{1}{h^{2}} \sin (\gamma h) \sin (\beta h) U_{i j}^{n} .
\end{aligned}
$$

We denote $r=\frac{\Delta t}{h^{2}}$.

After substituting the above difference operators in the scheme (21), we obtain the amplification factor

$$
\begin{aligned}
\xi= & \frac{-2 b r \sin (\gamma h) \sin (\beta h)}{(1-\operatorname{ar}(\cos (\beta h)-1))(1-\operatorname{cr}(\cos (\gamma h)-1))} \\
& +\frac{(1+\operatorname{ar}(\cos (\beta h)-1))(1+\operatorname{cr}(\cos (\gamma h-1)))}{(1-\operatorname{ar}(\cos (\beta h)-1))(1-\operatorname{cr}(\cos (\gamma h)-1))} .
\end{aligned}
$$

The scheme is stable if the amplification factor $\xi$ satisfies $|\xi| \leq 1$. The term $\xi$ can be written as

$$
\begin{aligned}
\xi= & \frac{-2 b r \sin (\gamma h) \sin (\beta h)}{\left(1+2 a r \sin ^{2}(\beta h / 2)\right)\left(1+2 c r \sin ^{2}(\gamma h / 2)\right)} \\
& +\frac{\left(1-2 a r \sin ^{2}(\beta h / 2)\right)\left(1-2 c r \sin ^{2}(\gamma h / 2)\right)}{\left(1+2 a r \sin ^{2}(\beta h / 2)\right)\left(1+2 c r \sin ^{2}(\gamma h / 2)\right)} .
\end{aligned}
$$

Next, we prove that $|\xi| \leq 1$. First, we need the following lemma.

Lemma 1 If $b^{2}<a c$, then the following inequality holds $4 a \sin ^{2}(\theta / 2)+4 c \sin ^{2}(\varphi / 2)-2|b \sin (\theta) \sin (\varphi)| \geq 0$,

$\forall \theta, \phi$.

Proof We have

$$
(\sqrt{a}|\sin (\theta / 2)|-\sqrt{c}|\sin (\varphi / 2)|)^{2} \geq 0 .
$$

Since $b^{2}<a c$, one has

$$
\begin{aligned}
& a \sin ^{2}(\theta / 2)+c \sin ^{2}(\varphi / 2) \\
& \quad \geq 2 \sqrt{a c}|\sin (\theta / 2) \sin (\varphi / 2)| \\
& \quad>2|b||\sin (\theta / 2) \sin (\varphi / 2)| .
\end{aligned}
$$

But one can see that

$|\sin (\theta / 2)| \geq \frac{1}{2}|\sin (\theta)|$.

Thus (28) and (29) provide the required inequality

$4 a \sin ^{2}(\theta / 2)+4 c \sin ^{2}(\varphi / 2)-2|b \sin (\theta) \sin (\varphi)| \geq 0$.

We need to show that $-1 \leq \xi \leq 1$. First, we show that $\xi \leq 1$.

$$
\begin{aligned}
\xi-1= & \left(\frac{-2 b r \sin (\gamma h) \sin (\beta h)}{\left(1+2 a r \sin ^{2}(\beta h / 2)\right)\left(1+2 c r \sin ^{2}(\gamma h / 2)\right)}\right. \\
& \left.+\frac{\left(1-2 a r \sin ^{2}(\beta h / 2)\right)\left(1-2 c r \sin ^{2}(\gamma h / 2)\right)}{\left(1+2 a r \sin ^{2}(\beta h / 2)\right)\left(1+2 c r \sin ^{2}(\gamma h / 2)\right)}\right) \\
& -1 \\
= & \frac{-4 a r \sin ^{2}(\beta h / 2)-4 c r \sin ^{2}(\gamma h / 2)}{\left(1+2 a r \sin ^{2}(\beta h / 2)\right)\left(1+2 c r \sin ^{2}(\gamma h / 2)\right)} \\
& -\frac{2 b r \sin (\gamma h) \sin (\beta h)}{\left(1+2 a r \sin ^{2}(\beta h / 2)\right)\left(1+2 c r \sin ^{2}(\gamma h / 2)\right)} .
\end{aligned}
$$

From the above lemma, we have

$-2 b r \sin (\gamma h) \sin (\beta h) \leq 4 a r \sin ^{2}(\beta h / 2)+4 c r \sin ^{2}(\gamma h / 2)$. 
We then conclude that $\xi \leq 1$. Next, we show that $\xi+$ $1 \geq 0$.

$$
\begin{aligned}
\xi+1= & \frac{-2 b r \sin (\gamma h) \sin (\beta h)}{\left(1+2 a r \sin ^{2}(\beta h / 2)\right)\left(1+2 c r \sin ^{2}(\gamma h / 2)\right)} \\
& +\frac{\left(1-2 a r \sin ^{2}(\beta h / 2)\right)\left(1-2 c r \sin ^{2}(\gamma h / 2)\right)}{\left(1+2 a r \sin ^{2}(\beta h / 2)\right)\left(1+2 c r \sin ^{2}(\gamma h / 2)\right)} \\
& +\frac{\left(1+2 a r \sin ^{2}(\beta h / 2)\right)\left(1+2 c r \sin ^{2}(\gamma h / 2)\right)}{\left(1+2 a r \sin ^{2}(\beta h / 2)\right)\left(1+2 c r \sin ^{2}(\gamma h / 2)\right)} .
\end{aligned}
$$

Thus,

$$
\begin{aligned}
\xi+1= & \frac{8 a c r^{2} \sin ^{2}(\beta h / 2) \sin ^{2}(\gamma h / 2)}{\left(1+2 a r \sin ^{2}(\beta h / 2)\right)\left(1+2 c r \sin ^{2}(\gamma h / 2)\right)} \\
& -\frac{2 b r \sin (\gamma h) \sin (\beta h)+2}{\left(1+2 a r \sin ^{2}(\beta h / 2)\right)\left(1+2 c r \sin ^{2}(\gamma h / 2)\right)} .
\end{aligned}
$$

In order to get $\xi+1 \geq 0$, we need to show that the numerator of the above expression is positive, i.e.

$$
\begin{aligned}
& 8 a c r^{2} \sin ^{2}(\beta h / 2) \sin ^{2}(\gamma h / 2) \\
& \quad-2 b r \sin (\gamma h) \sin (\beta h)+2 \geq 0 .
\end{aligned}
$$

Let us analyze the discriminant of this quadratic equation:

$$
\begin{aligned}
\Delta= & r^{2}\left[b^{2} \sin ^{2}(\beta h) \sin ^{2}(\gamma h)\right. \\
& \left.-16 a^{2} c^{2} \sin ^{2}(\beta h / 2) \sin ^{2}(\gamma h / 2)\right] .
\end{aligned}
$$

Next, we use the relation $b^{2}<a c$, and get

$$
\begin{aligned}
\Delta< & r^{2}(a c)^{2} \sin ^{2}(\beta h)\left[\sin ^{2}(\gamma h)-16 \sin ^{2}(\gamma h / 2)\right] \\
\leq & 16 r^{2}(a c)^{2} \sin ^{2}(\beta h / 2) \sin ^{2}(\gamma h / 2) \\
& \times\left(\cos ^{2}(\beta h / 2) \cos ^{2}(\gamma h / 2)-1\right) \leq 0 .
\end{aligned}
$$

This implies $\Delta<0$, i.e. $-1 \leq \xi$.

We obtained that $-1 \leq \xi \leq 1$. Thereby, we showed that the modulus of the amplification term is bounded by 1 independently of $r$. Thus, we conclude that for the simple case of constant coefficients, the semi-implicit approximation based on Crank-Nicolson scheme is unconditionally stable.

We note that for the AOS scheme, one can find time-steps and diffusion operators for which the scheme is unstable in the linear case. The AOS method does remain stable for the Beltrami flow in practice, with maximally stable time-steps similar to those allowed by the LOD scheme.

\subsection{LOD/AOS Scheme for the Beltrami-Based Denoising}

The splitting scheme in the presence of a fidelity term requires a slight modification that we detail below. In this case we solve for each channel the equation

$U_{t}^{a}=-\frac{2 \lambda}{\sqrt{g}}\left(U^{a}-F^{a}\right)+\Delta_{g} U^{a}$,

with von-Neumann boundary condition and the initial condition

$U^{a}(x, 0)=F^{a}(x)$.

The Crank-Nicolson scheme approximating (33) is

$$
\begin{aligned}
& \left(U^{a}\right)^{n+1} \\
& =\left(I-\frac{\Delta t}{2} \sum_{l=1}^{2} \bar{A}_{l l}^{n}+2 \Delta t \frac{\lambda}{\sqrt{g^{n}}} I\right)^{-1} \\
& \times\left[\left(\left(I+\frac{\Delta t}{2} \bar{A}_{11}^{n}\right)\left(I+\frac{\Delta t}{2} \bar{A}_{22}^{n}\right)\right.\right. \\
& \left.\left.+\Delta t \sum_{q=1}^{2} \sum_{r \neq q} \bar{A}_{q r}^{n}\right)\left(U^{a}\right)^{n}+2 \Delta t F^{a} \frac{\lambda}{\sqrt{g^{n}}}\right] .
\end{aligned}
$$

It is possible to use LOD/AOS approximations for the inverse of the matrix in the above scheme. However, we would like to treat the fidelity term in a special way. When the term $\lambda / \sqrt{g^{n}}$ is large, we find that the scheme proposed below possesses better stability properties.

We now describe the details for treating the fidelity term for our Crank-Nicolson scheme. Dividing the numerator and the denominator by the matrix

$S^{n}=\left(1+2 \Delta t \frac{\lambda}{\sqrt{g^{n}}}\right) I$

and rearranging terms, we get

$\left(U^{a}\right)^{n+1}$

$$
\begin{aligned}
& =\left(I-\frac{\Delta t}{2}\left(S^{n}\right)^{-1} \sum_{l=1}^{2} \bar{A}_{l l}^{n}\right)^{-1} \\
& \times\left[( S ^ { n } ) ^ { - 1 } \left(\left(I+\frac{\Delta t}{2} \bar{A}_{11}^{n}\right)\left(I+\frac{\Delta t}{2} \bar{A}_{22}^{n}\right)\right.\right. \\
& \left.+\Delta t \sum_{q=1}^{2} \sum_{r \neq q} \bar{A}_{q r}^{n}\right)\left(U^{a}\right)^{n} \\
& \left.+2\left(S^{n}\right)^{-1} \Delta t F^{a} \frac{\lambda}{\sqrt{g^{n}}}\right] .
\end{aligned}
$$


Approximating the semi-implicit scheme based on the LOD-splitting, we have

$$
\begin{aligned}
& \left(U^{a}\right)^{n+1} \\
& =\left(I-\frac{1}{2} \Delta t\left(S^{n}\right)^{-1} \bar{A}_{22}^{n}\right)^{-1}\left(I-\frac{1}{2} \Delta t\left(S^{n}\right)^{-1} \bar{A}_{11}^{n}\right)^{-1} \\
& \quad \times\left[( S ^ { n } ) ^ { - 1 } \left(\left(I+\frac{\Delta t}{2} \bar{A}_{11}^{n}\right)\left(I+\frac{\Delta t}{2} \bar{A}_{22}^{n}\right)\right.\right. \\
& \left.+\Delta t \sum_{q=1}^{2} \sum_{r \neq q} \bar{A}_{q r}^{n}\right)\left(U^{a}\right)^{n} \\
& \left.+2\left(S^{n}\right)^{-1} \Delta t F^{a} \frac{\lambda}{\sqrt{g^{n}}}\right] .
\end{aligned}
$$

While using the AOS splitting, the Crank-Nicolson scheme reads

$$
\begin{aligned}
& \left(U^{a}\right)^{n+1} \\
& =\frac{1}{2}\left(\left(I-\Delta t\left(S^{n}\right)^{-1} \bar{A}_{11}^{n}\right)^{-1}+\left(I-\Delta t\left(S^{n}\right)^{-1} \bar{A}_{22}^{n}\right)^{-1}\right) \\
& \quad \times\left[( S ^ { n } ) ^ { - 1 } \left(\left(I+\frac{\Delta t}{2} \bar{A}_{11}^{n}\right)\left(I+\frac{\Delta t}{2} \bar{A}_{22}^{n}\right)\right.\right. \\
& \left.\left.+\Delta t \sum_{q=1}^{2} \sum_{r \neq q} \bar{A}_{q r}^{n}\right)\left(U^{a}\right)^{n}+2\left(S^{n}\right)^{-1} \Delta t F^{a} \frac{\lambda}{\sqrt{g^{n}}}\right] .
\end{aligned}
$$

The above schemes are efficient since at each time step we only need to perform a tridiagonal matrix inversion.

Below we provide a stability analysis for the linear case when a fidelity term is present.

The equation we need to discretize is:

$$
U_{t}=a U_{x x}+2 b U_{x y}+c U_{y y}+2 \lambda(f-U),
$$

with constants $a, b, c$ satisfying $b^{2}<a c$ and $\lambda>0$.

Denote $Q=\frac{1}{1+2 \lambda \Delta t}$.

The Crank-Nicolson scheme with LOD splitting for approximating (38) can be written as:

$$
\begin{aligned}
U^{n+1}= & \left(\left(I-a \frac{\Delta t}{2} Q \partial_{x x}\right)^{-1}\left(I-c \frac{\Delta t}{2} Q \partial_{y y}\right)^{-1}\right) \\
& \times\left[Q \left(\left(I+\frac{\Delta t}{2} a \partial_{x x}\right)\left(I+\frac{\Delta t}{2} c \partial_{y y}\right)\right.\right. \\
& \left.\left.+2 \Delta t b \partial_{x y}\right) U^{n}+2 Q \Delta t \lambda f\right]
\end{aligned}
$$

Consider a solution of the difference scheme in the form

$U_{i j}^{n}=M e^{\alpha n \Delta t} e^{\sqrt{-1} i \beta h} e^{\sqrt{-1} j \gamma h}$,

where $\beta$ and $\gamma$ are constant wave numbers.

For establishing stability, we use the definition of stability in the Lax-Richtmeyer sense. Therefore we require

$\left|e^{\alpha \Delta t}\right| \leq 1+K \Delta t$

where $K$ is a constant.

Indeed if (40) is satisfied, it follows that

$$
\begin{aligned}
|U|^{n} & \leq\left|e^{\alpha n \Delta t}\right| \leq(1+K \Delta t)^{n} \\
& \leq e^{K n \Delta t}=e^{t_{n} K}<e^{T K},
\end{aligned}
$$

for all $t_{n}<T$, which reveals the stability of the solution.

Lemma 2 The amplification factor for the LOD scheme satisfies

$\left|e^{\alpha \Delta t}\right| \leq 1+2 \frac{\lambda}{M}|f| \Delta t$.

Proof The required inequality is clear if $\left|e^{\alpha \Delta t}\right| \leq 1$. We then assume

$\left|e^{\alpha \Delta t}\right|>1$

Replacing the relations (24), (25), (26) into our scheme leads to

$e^{\alpha \Delta t}=\xi_{1}+\xi_{0} \Delta t$

where

$$
\begin{aligned}
\xi_{1}= & \frac{Q[-2 b r \sin (\gamma h) \sin (\beta h)}{(1-\operatorname{ar} Q(\cos (\beta h)-1))((1-\operatorname{cr} Q(\cos (\gamma h)-1)))} \\
& +\frac{(1+\operatorname{ar}(\cos (\beta h)-1))(1+\operatorname{cr}(\cos (\gamma h)-1))]}{(1-\operatorname{ar} Q(\cos (\beta h)-1))((1-\operatorname{cr} Q(\cos (\gamma h)-1)))}
\end{aligned}
$$

and

$$
\begin{aligned}
\xi_{0}= & \frac{1}{1+2 \Delta t} \\
& \times \frac{2 \lambda f}{(1-\operatorname{ar} Q(\cos (\beta h)-1))((1-\operatorname{cr} Q(\cos (\gamma h)-1))) U^{n}} .
\end{aligned}
$$

We first show that $\left|\xi_{1}\right| \leq 1$. We have

$\left|\xi_{1}\right| \leq \frac{\left|-2 b r \sin (\gamma h) \sin (\beta h)+\left(1-2 a r \sin ^{2}\left(\frac{\beta h}{2}\right)\right)\left(1-2 c r \sin ^{2}\left(\frac{\gamma h}{2}\right)\right)\right|}{\left(1+2 \lambda \Delta t+2 a r \sin ^{2}\left(\frac{\beta h}{2}\right)\right)\left(1+2 \lambda \Delta t+2 c r \sin ^{2}\left(\frac{\gamma h}{2}\right)\right)}$ 
Using the computation of the amplification factor $\xi$ for the scale space case (see (27)), we get

$\left|\xi_{1}\right| \leq|\xi| \leq 1$

for all $\lambda>0$.

Next, we analyze the term $\xi_{0}$.

$\left|\xi_{0}\right|=\frac{2 Q \lambda|f| /\left|U^{n}\right|}{\left(1+2 \lambda \Delta t+2 \operatorname{ar} \sin ^{2}\left(\frac{\beta h}{2}\right)\right)\left(1+2 \lambda \Delta t+2 c r \sin ^{2}\left(\frac{\gamma h}{2}\right)\right)}$.
From assumption (42) we get $\left|e^{\alpha n \Delta t}\right|>1$ for all $n$, which by means of (39), leads to $\left|U^{n}\right|>M$. Moreover using $Q=$ $\frac{1}{1+2 \lambda \Delta t}<1$ we get:

$\left|\xi_{0}\right| \leq \frac{2}{M} \lambda|f|$

From (47) and (49) we obtain the needed inequality (41).
Fig. 1 Top row, left: The original image which contains JPEG artifacts. Right: Results of the LOD splitting scheme with $\Delta t=1$, after 1 iteration. Middle row, left and right: Results of the LOD splitting scheme with $\Delta t=1$, after 2 and 4 iterations, respectively $\left(\beta=\sqrt{10^{3}}, \lambda=0\right)$. Bottom row, left: a close-up of the original image. Right: a close-up of the resulting image after 4 iterations
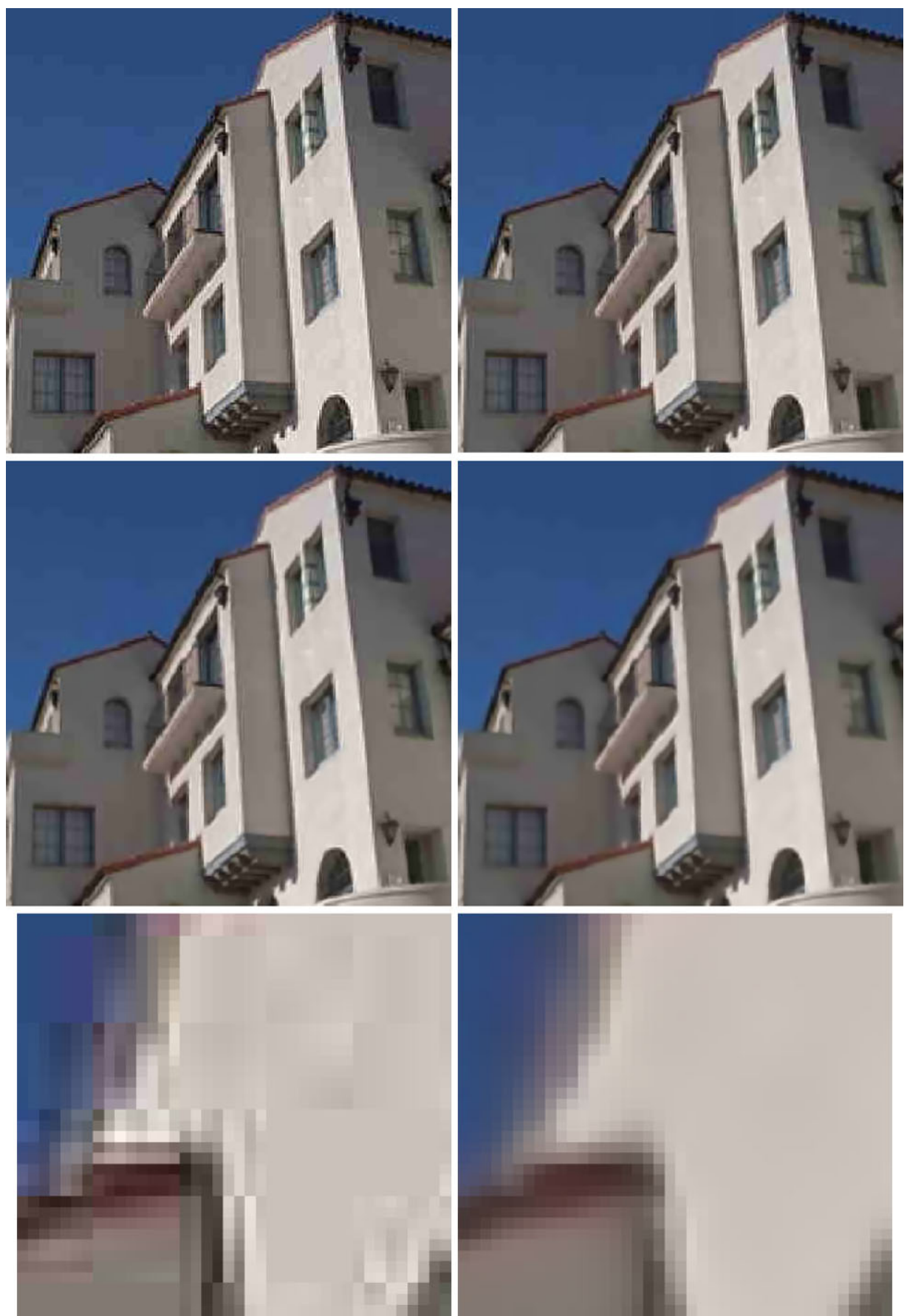
Fig. 2 The different image channels of an image patch taken from the images in Fig. 1. Left to right, top to bottom: An image patch before denoising, its different color channels, the denoised image, and the denoised color channels. The color arrows indicate the direction of the gradient in the various color channels
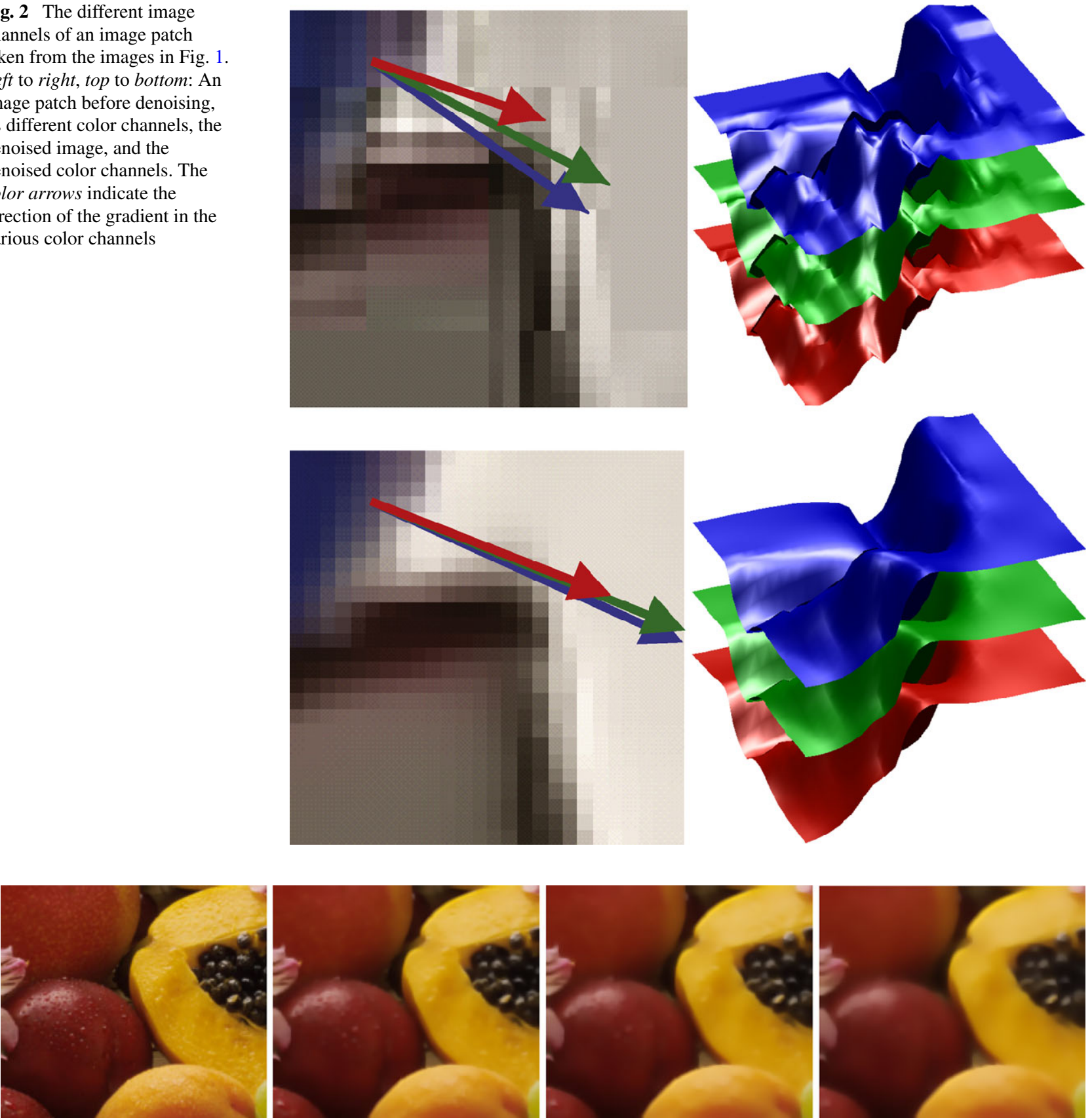

Fig. 3 Beltrami scale-space computed using the LOD splitting scheme. Top left: The original image. Top right: Results of the LOD splitting scheme with $\Delta t=10^{-1}$, after 20 iterations. Bottom left: Results after 40 iterations. Bottom right: Results after 80 iterations $\left(\beta=\sqrt{10^{3}}, \lambda=0\right)$

We thus conclude that also in the denoising case with constant coefficients, the semi-implicit LOD approximation based on the Crank-Nicolson scheme is unconditionally stable.

\section{Experimental Results}

We proceed to demonstrate experimentally the stability, accuracy, and efficiency of the LOD and AOS splitting schemes for the Beltrami color flow. In Figs. 1-3 we show the results of the Beltrami flow, implemented by employing the LOD splitting scheme for approximating (5). This is performed both for denoising purposes and for scale-space analysis.

Next we illustrate the use of the splitting schemes in the case where the functional involves a fidelity term. A noisy image as well as the reference denoising result, based on the explicit scheme, are shown in Fig. 4. In Fig. 5 we show the 


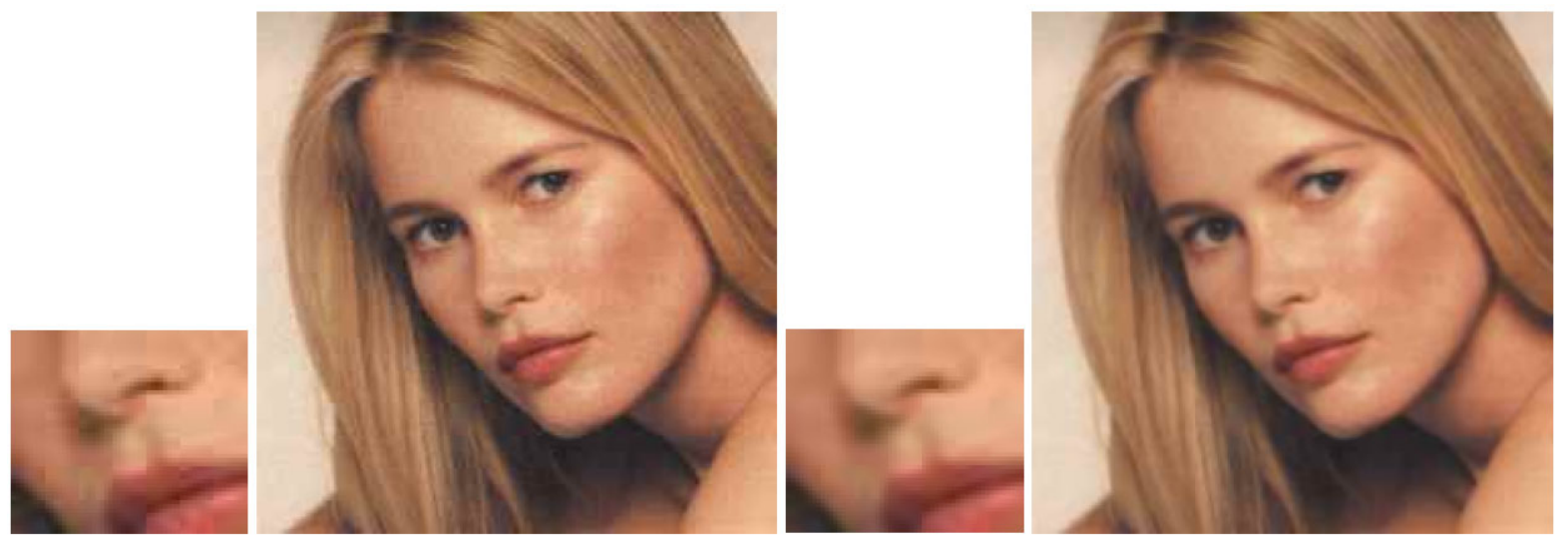

Fig. 4 Left: An image with artifacts resulting from lossy compression. Right: The reference image. Beltrami-based denoising by explicit scheme, run with 4000 explicit iterations, $\Delta t=0.0005, \lambda=1, \beta=\sqrt{5 \times 10^{4}}$

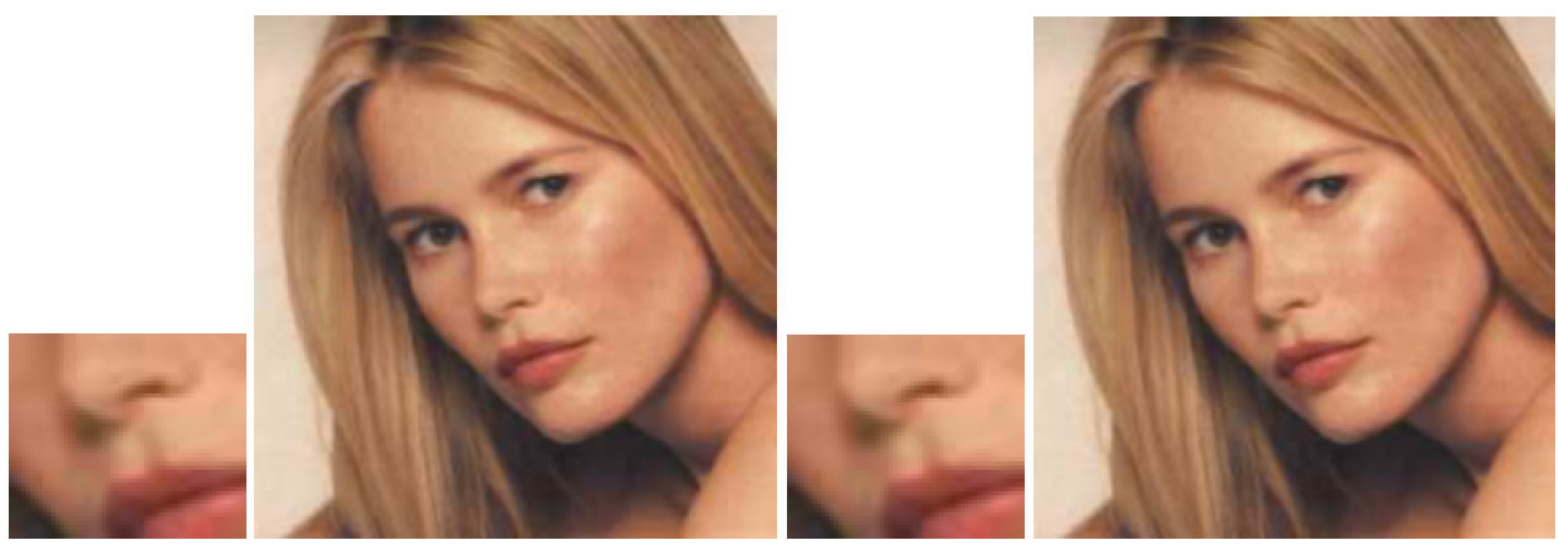

Fig. 5 Comparison of the images obtained using LOD and AOS schemes. Left: Denoising by LOD. Right: Denoising by AOS. ( $\lambda=1, \Delta t=0.02$, $\left.\beta=\sqrt{5 \times 10^{4}}\right)$

Fig. 6 Left: An image after the introduction of channel-wise salt and pepper noise. Right: The image after Beltrami-based denoising, using 100 LOD iterations, $\Delta t=0.5, \lambda=10^{-2}$, $\beta=\sqrt{2 \times 10^{4}}$
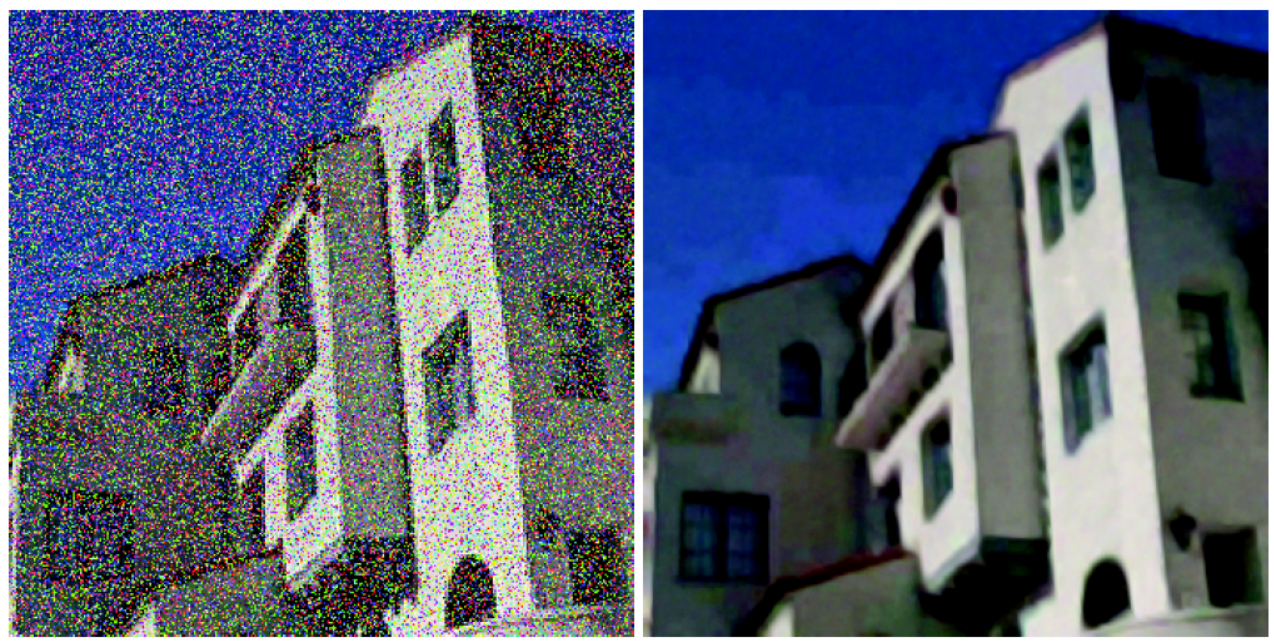

result of the AOS and LOD splitting schemes. Note that the visual results obtained by the two schemes are similar to the reference image. The effectiveness of the denoising effect is particularly pronounced where the noisy image deviates strongly from what is expected by the Lambertian image formation model.

This can be seen, for example, on decorrelated salt and pepper noise, as demonstrated in Fig. 6. For the purpose of 


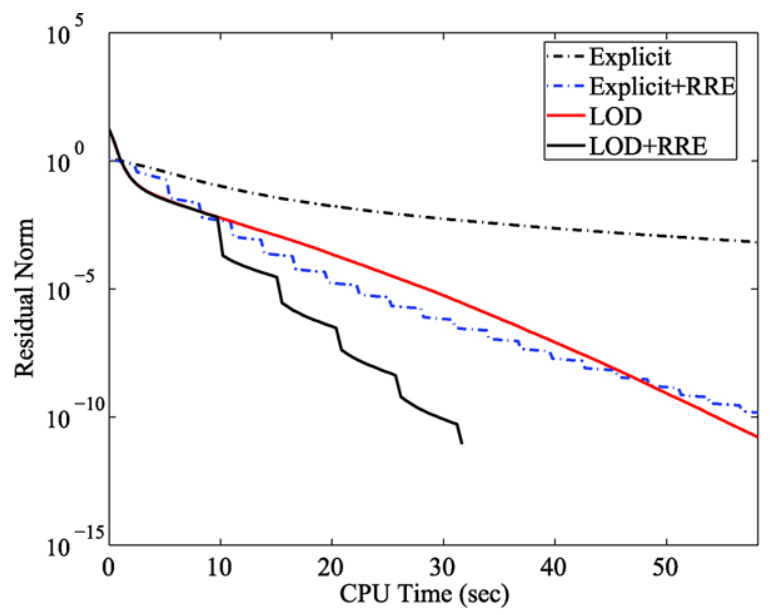

(a)

Fig. 7 Left: Graph of the residuals (explicit, LOD, explicit + RRE and LOD + RRE) versus CPU times. Parameters: $\Delta t=0.2$ for the explicit scheme, $\Delta t=3$ for LOD, $\lambda=0.5, \beta=\sqrt{3 \times 10^{3}}$. Right: Comparison

Fig. 6, a robust fidelity term was added, along the lines suggested in [3]. Specifically, the descent equation was

$U_{t}^{a}=-\frac{\lambda}{\sqrt{g}} \frac{\left(U^{a}-F^{a}\right)}{\sqrt{\left(U^{a}-F^{a}\right)^{2}+\epsilon}}+\Delta_{g} U^{a}$,

where $\lambda$ controls the regularization as before, and $\epsilon$ is a small constant, taken to be $10^{-3}$ in our case.

\subsection{RRE Technique for Acceleration of the LOD Splitting Scheme}

In [7] vector extrapolation was applied in order to speed up the slow convergence of the explicit schemes for the Beltrami color flow. In the experiments below we demonstrate how the RRE extrapolation technique can also be used to accelerate the convergence of implicit schemes. Figure 7(a) shows that the RRE method accelerates the LOD scheme. A comparison is also given to the convergence rate achieved by the method of [7].

In Fig. 7(b) the accuracy of the LOD based scheme, as well as the LOD based scheme combined with RRE is considered with respect to different time steps, in order to observe the rate of acceleration of the RRE extrapolation scheme. The reference image for the computation of the MSE error of the schemes is the image obtained by the explicit scheme with a small time-step. Figure 7(a) indicates how the RRE extrapolation scheme leads to a better acceleration rate when using a relative small time step. The time-steps used are considerably larger than the time steps needed for maintaining the stability of the explicit scheme (the largest possible time step preserving numerical stability for this example was $\Delta t=0.2$ ). Even for these relatively

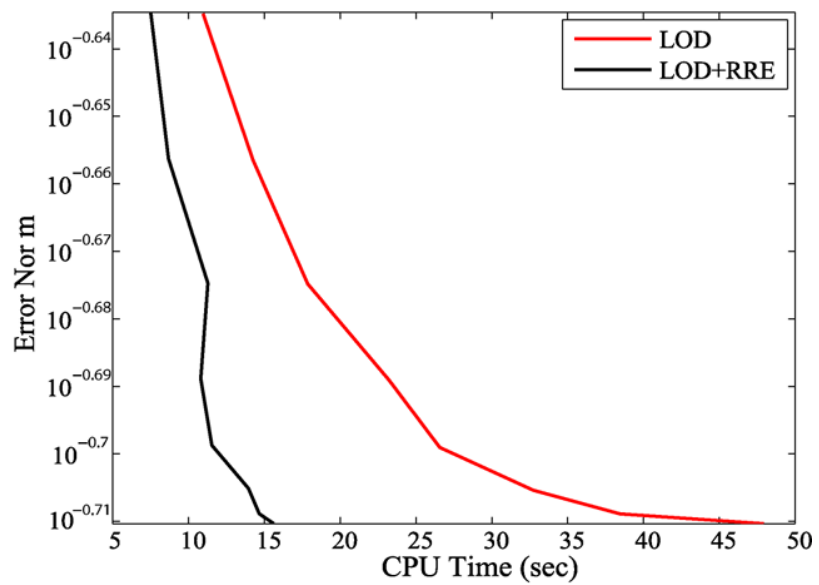

(b)

of CPU times for LOD and accelerated LOD (for the denoising case). CPU time versus error norm. Time steps: $\Delta t=0.34,0.42,0.52,0.66$, $0.82,1.02,1.28,1.6 ., \lambda=0.1, \beta=\sqrt{3 \times 10^{3}}$

large time steps, however, the RRE method results in a significant speedup without compromising the accuracy of the scheme.

\subsection{Beltrami Deblurring of Images Using Splitting Schemes}

Another common usage for nonlinear regularization is in deblurring images. The Euler-Lagrange equations characterizing the deblurring of an image blurred using a convolution kernel $k$ is

$U_{t}^{a}=\Delta_{g} U^{a}-2 \sum_{a} \bar{k} *\left(k * U^{a}-U_{0}^{a}\right)$.

In the deblurring case, we split the operator $\bar{k} * k$ operating on $U^{a}$ by taking the center element of its influence on the image at time $\left(n+\frac{1}{2}\right) \Delta t$, and using an inverted matrix $S^{n}$ similar to the one described in (35). Here we make the assumption that the deblurring operator's energy is largely centered around the middle pixel. While this leaves an explicit part of the kernel which may limit the time steps used, in the experiments made by us this limitation surfaced at step sizes at which operator accuracy was already a limiting factor. These time steps are furthermore significantly larger than the ones possible with the explicit deblurring scheme. In Fig. 8 we show Beltrami-regularized deblurring, for both Gaussian blur and (location independent) motion blur deblurring.

\section{Conclusions}

Due to its anisotropy and non-separability, no implicit scheme, nor operator splitting based scheme was so far 
Fig. 8 Left-to-right, top-to-bottom: (ab) An image blurred by Gaussian filter with standard deviation $\sigma=3$,

(b) the same image after deblurring using LOD-based Beltrami deblurring, (c) an image blurred by a narrow Gaussian filter similar to motion blur, (d) the same image after deblurring. Parameters: $\Delta t=0.05$, scheme, $\lambda=5 \times 10^{4}, \beta=\frac{1}{100}$

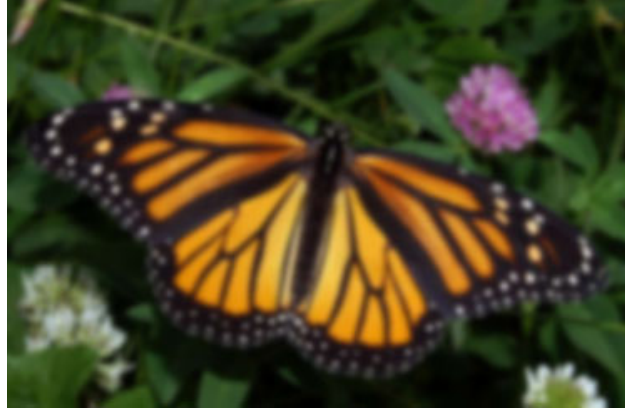

(a)

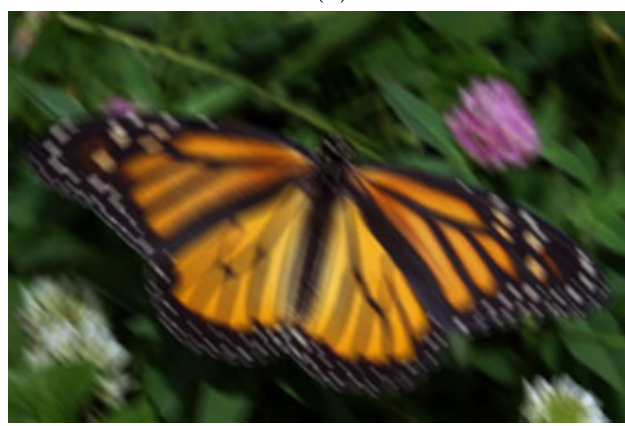

(c)

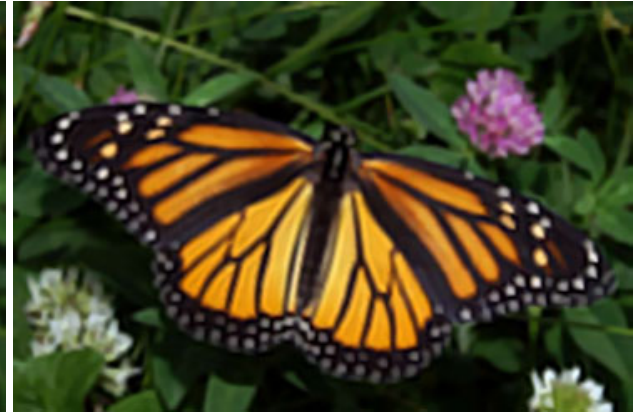

(b)

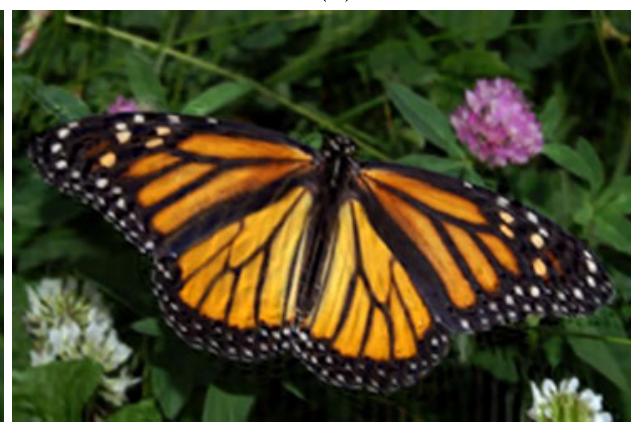

(d) introduced for the partial differential equations that describe the Beltrami color flow. In this paper we propose a semi-implicit splitting scheme based on LOD/AOS for the anisotropic Beltrami operator. The spatial mixed derivatives are discretized explicitly at time step $n \Delta t$, while the nonmixed derivatives are approximated using the average of the two time levels $n \Delta t$ and $(n+1) \Delta t$.

We provide a von-Neumann stability analysis of the LOD-based scheme, which is valid in the simple case where the coefficients of the equation are constants. In the more general nonlinear case, the stability of the splitting is empirically tested in applications such as Beltrami-based scalespace and Beltrami-based denoising, which display a stable behavior. In order to further accelerate the convergence of the splitting schemes, the RRE vector extrapolation technique is employed.

Acknowledgements We thank Prof. Avram Sidi for interesting discussions.

Open Access This article is distributed under the terms of the Creative Commons Attribution Noncommercial License which permits any noncommercial use, distribution, and reproduction in any medium, provided the original author(s) and source are credited.

\section{References}

1. Andreev, V.B.: Alternating direction methods for parabolic equations in two space dimensions with mixed derivatives. Ž. Vyčisl. Mat. Mat. Fiz. 7(2), 312-321 (1967)
2. Aurich, V., Weule, J.: Non-linear Gaussian filters performing edge preserving diffusion. In: Mustererkennung 1995, 17. DAGMSymposium, pp. 538-545. Springer, London (1995)

3. Bar, L., Brook, A., Sochen, N., Kiryati, N.: Color image deblurring with impulsive noise. In: Proceedings of the International Conference on Variational, Geometry and Level Sets Methods in Computer Vision, vol. 3752, pp. 49-60 (2005)

4. Barash, D.: A fundamental relationship between bilateral filtering, adaptive smoothing and the nonlinear diffusion equation. IEEE Trans. Image Process. 24(6), 844-847 (2002)

5. Barash, D., Schlick, T., Israeli, M., Kimmel, R.: Multiplicative operator splittings in nonlinear diffusion: from spatial splitting to multiple timesteps. J. Math. Imaging Vis. 19(16), 33-48 (2003)

6. Buades, A., Coll, B., Morel, J.M.: A review of image denoising algorithms, with a new one. Multiscale Model. Simul. 4(2), 490530 (2005)

7. Dascal, L., Rosman, G., Kimmel, R.: Efficient Beltrami filtering of color images via vector extrapolation. In: Scale Space and Variational Methods in Computer Vision. Lecture Notes on Computer Science, vol. 4485, pp. 92-103. Springer, Berlin (2007)

8. Dascal, L., Rosman, G., Tai, X.C., Kimmel, R.: On semi-implicit splitting schemes for the Beltrami color flow. In: Scale Space and Variational Methods in Computer Vision. Lecture Notes on Computer Science, vol. 5567, pp. 259-270. Springer, Berlin (2009)

9. Eddy, R.: Extrapolating to the limit of a vector sequence. In: Wang, P. (ed.) Information Linkage Between Applied Mathematics and Industry, pp. 387-396. Academic Press, New York (1979)

10. Elad, M.: On the bilateral filter and ways to improve it. IEEE Trans. Image Process. 11(10), 1141-1151 (2002)

11. Kimmel, R., Malladi, R., Sochen, N.: Images as embedding maps and minimal surfaces: Movies, color, texture, and volumetric medical images. Int. J. Comput. Vis. 39(2), 111-129 (2000)

12. Lu, T., Neittaanmäki, P., Tai, X.C.: A parallel splitting up method and its application to Navier-Stokes equations. Appl. Math. Lett. 4(2), 25-29 (1991)

13. Lu, T., Neittaanmäki, P., Tai, X.C.: A parallel splitting up method for partial differential equations and its application to NavierStokes equations. RAIRO. Anal. Numér. 26(6), 673-708 (1992) 
14. Mahmoudi, M., Sapiro, G.: Fast image and video denoising via nonlocal means of similar neighborhoods. IEEE Signal Process. Lett. 12, 839-842 (2005)

15. Marchuk, G.I.: Splitting and alternating direction methods. In: Handbook of Numerical Analysis, vol. I, pp. 197-462. NorthHolland, Amsterdam (1990)

16. Mckee, S., Mitchell, A.R.: Alternating direction methods for parabolic equations in three space dimensions with mixed derivatives. Comput. J. 14(3), 25-30 (1971)

17. Mešina, M.: Convergence acceleration for the iterative solution of the equations $X=A X+f$. Comput. Methods Appl. Mech. Eng. 10, 165-173 (1977)

18. Paris, S., Durand, F.: A fast approximation of the bilateral filter using a signal processing approach. In: Leonardis, A., Bischof, H., Pinz, A. (eds.) European Conf. on Comp. Vision. Lecture Notes on Computer Science, vol. 3951, pp. 568-580. Springer, Berlin (2006)

19. Peaceman, D.W., Rachford, H.H.: The numerical solution of parabolic and elliptic differential equations. J. Soc. Ind. Appl. Math. 3, 28-41 (1955)

20. Polyakov, A.M.: Quantum geometry of bosonic strings. Phys. Lett. B 103, 207-210 (1981)

21. Rudin, L., Osher, S., Fatemi, E.: Non-linear total variation based noise removal algorithms. Physica D 60, 259-268 (1992)

22. Smith, S.M., Brady, J.: SUSAN—a new approach to low level image processing. Int. J. Comput. Vis. 23, 45-78 (1997)

23. Sochen, N., Kimmel, R., Bruckstein, A.M.: Diffusions and confusions in signal and image processing. J. Math. Imaging Vis. 14(3), 195-209 (2001)

24. Sochen, N., Kimmel, R., Maladi, R.: From high energy physics to low level vision. In: ter Haar Romeny, B.M., Florack, L., Koenderink, J.J., Viergever, M.A. (eds.) Scale-Space Theory in Computer Vision. Lecture Notes on Computer Science, vol. 1252, pp. 236-247. Springer, Berlin (1997)

25. Sochen, N., Kimmel, R., Maladi, R.: A general framework for low level vision. IEEE Trans. Image Process. 7, 310-318 (1998)

26. Spira, A., Kimmel, R., Sochen, N.A.: A short-time Beltrami kernel for smoothing images and manifolds. IEEE Trans. Image Process. 16(6), 1628-1636 (2007)

27. Tomasi, C., Manduchi, R.: Bilateral filtering for gray and color images. In: Proceedings of IEEE International Conference on Computer Vision, pp. 836-846 (1998)

28. Weickert, J.: Coherence-enhancing diffusion filtering. Int. J. Comput. Vis. 31(2/3), 111-127 (1999)

29. Weickert, J., Romeny, B.M.T.H., Viergever, M.A.: Efficient and reliable schemes for nonlinear diffusion filtering. IEEE Trans. Image Process. 7(3), 398-410 (1998)

30. Yanenko, N.N.: About implicit difference methods of the calculation of the multidimensional equation of thermal conductivity. Izv. Vysš. Učebn. Zaved., Mat. 23(4), 148-157 (1961)

31. Yanenko, N.N.: The Method of Fractional Steps. The Solution of Problems of Mathematical Physics in Several Variables. Springer, New York (1971)

32. Yezzi, A.J.: Modified curvature motion for image smoothing and enhancement. IEEE Trans. Image Process. 7(3), 345-352 (1998)

33. Yoshizawa, S., Belyaev, A., Seidel, H.P.: Smoothing by example: mesh denoising by averaging with similarity-based weights. In: Proceedings of International Conference on Shape Modelling and Applications, pp. 38-44 (2006)

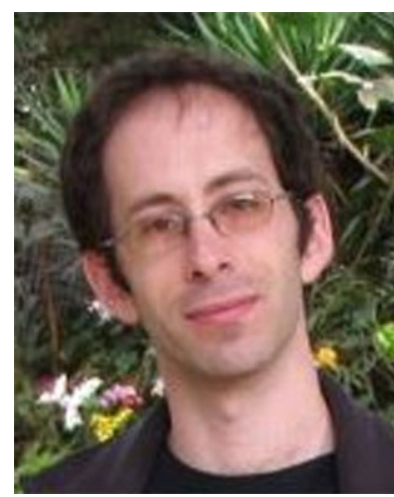

Guy Rosman is currently pursuing his $\mathrm{PhD}$ in the Computer Science Department, at the Technion, Israel. He graduated in 2004 his BSc Summa Cum Laude at the Technion, and in 2008 his MSc, Cum Laude, at the Technion, in the Computer Science Department. During his studies, he worked at IBM's Haifa Research Labs, and in Rafael Advanced Defense Systems LTD as an algorithm developer, as well as an algorithm developer in Medicvision Imaging Solutions LTD from 2007. His research interests include variational and PDE based image processing, as well as algorithms for motion and structure estimation.

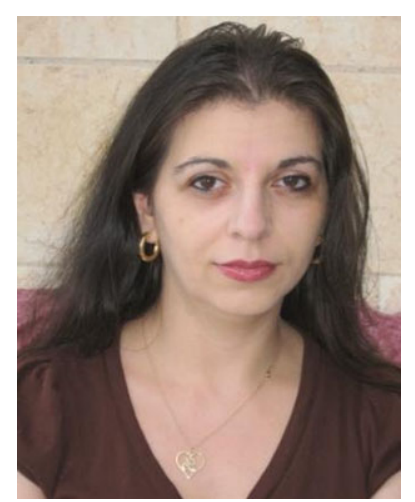

Lorina Dascal received the B.Sc. degree in Mathematics (Summa Cum Laude) from University of Iassy, Romania in 1998, the M.Sc. (Magna Cum Laude) in Applied Mathematics from Tel-Aviv University, Israel in 2001 and the Ph.D. in Applied Mathematics from TelAviv University in 2006. From 2006 to 2008 she was a postdoc in the Department of Computer Science at Technion-Israel Institute of Technology. For the last couple of years she has been employed at Mediguide St. Jude Medical, Haifa, Israel, where she holds the position of algorithm developer for imaging products. Her research interests include applications of variational methods and partial differential equations in computer vision, medical imaging, image processing and analysis.

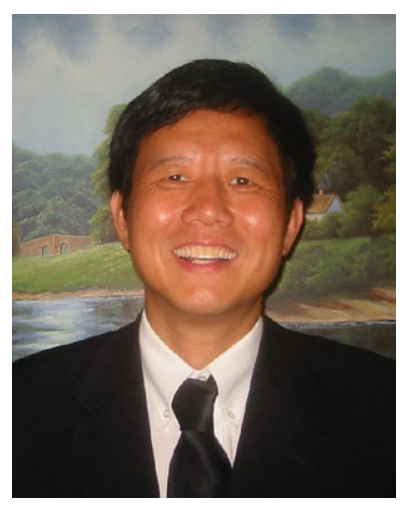

Xue-Cheng Tai received the Licenciate degree in 1989 and the Ph.D. degree in 1991 in applied mathematics from Jyvaskalya University, Finland. The subject of his Ph.D. dissertation was on inverse problems and parallel computing. After holding several research positions in Europe, he was employed as an Associate Professor in 1994 at the University of Bergen, Bergen, Norway and as a Professor since 1997. He has also worked as a part time Senior Scientist at the private company Rogaland Research. He is now a member of the Center for Mathematics for Applications in Oslo, Norway, and a member of the Center of Integrated Petroleum Research, Bergen, Norway. His research interests include Numerical PDE for image processing, multigrid and domain decomposition methods, iterative methods for linear and nonlinear PDE problems, and parallel computing. He has educated numerous M.S. and Ph.D. students and has published more than 60 scientific papers. He has been a Reviewer and Editor for several international journals. 


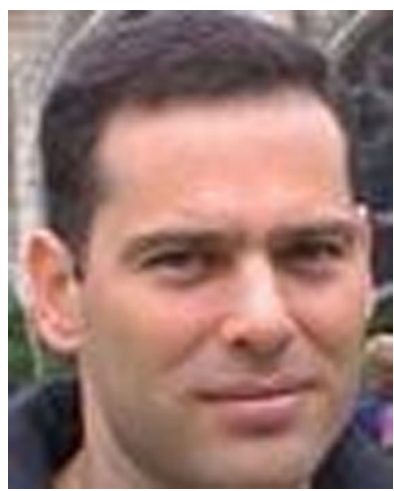

Ron Kimmel is a researcher in the areas of computer vision, image processing, and computer graphics. He is a tenured Professor at the Computer Science Dep., Technion. $\mathrm{He}$ is a Technion graduate (PhD/DSc 1995), spent his postdoctoral years (1995-1998) at Berkeley, and was a visiting professor at Stanford (2003-2004).

Kimmel has published over a hundred and fifty articles and papers in scientific journals and conferences. $\mathrm{He}$ is on the editorial board of International Journal of Computer Vision (IJCV), SIAM Journal of Imaging Sciences, Journal of Mathematical Imaging and Vision (JMIV), and was on the editorial board of IEEE Transactions on Image Process- ing (TIP). He served as a general co-chair of conferences like ScaleSpace Theories in Computer Vision, SIAM conf. on imaging, and the First Workshop on Non-Rigid Shape Analysis and Deformable Image Alignment (NORDIA-2008), as well as the Tenth Asian Conference on Computer Vision (ACCV-2010).

Ron Kimmel is the author of Numerical Geometry of Images, published by Springer in 2003 and co-author of Numerical Geometry of Non-Rigid Shapes, published by Springer in October 2008. Prof. Kimmel is the recipient of numerous prizes and decorations, including: IEEE Fellow (2009), the Hershel Rich Technion Innovation Award (twice), the Henry Taub Prize for Excellence in Research, as well as Alon, HTI, Wolf, Gutwirth, Ollendorff, and Jury fellowships. He was a consultant to HP research Labs (1998-2000), and to Net2Wireless/Jigami research (2000-2001). He was on the advisory board of MediGuide (biomedical imaging, until it was acquired) and a co-founder of Agileye Technologies, and BBKTechnologies. 